\title{
Systemic juvenile xanthogranuloma: a case of spontaneous regression of intramedullary spinal cord, cerebral, and cutaneous lesions
}

\author{
Anne Morice, MD, ${ }^{1,2}$ Sylvie Fraitag, MD, ${ }^{3}$ Catherine Miquel, MD, ${ }^{4}$ Christian Sainte Rose, MD, $\mathrm{PhD},{ }^{2}$ \\ and Stéphanie Puget, MD, PhD²
}

\begin{abstract}
Departments of ${ }^{1}$ Pediatric Plastic and Maxillofacial Surgery, ${ }^{2}$ Neurosurgery, and ${ }^{3}$ Pathology, Hôpital Necker, Université Paris Descartes, Sorbonne Paris Cité; and ${ }^{4}$ Department of Pathology, Saint-Louis Hospital, Université Paris Diderot, Sorbonne Paris Cité, Paris, France
\end{abstract}

\begin{abstract}
Juvenile xanthogranuloma (JXG) is a rare disease that belongs to the non-Langerhans cell histiocytoses. It presents a wide clinical spectrum, usually occurs before 5 years of age, and is commonly confined to the skin; however, it can affect multiple sites, including the nervous system, and can lead to severe disorders. Although JXG is a benign disease that usually regresses spontaneously, several curative treatments have been proposed in cases of organ involvement. Treatment options include corticosteroids, chemotherapy, and radiotherapy; however, these can have severe, long-term adverse effects in children.

The authors here describe the first case of spontaneous resolution of an intramedullary spinal cord lesion of JXG associated with cerebral and cutaneous lesions in a young boy with 9 years of follow-up. The initial neurological symptoms resolved without any surgical or medical treatment. This case shows that extracutaneous lesions of JXG, including those with intramedullary spinal cord involvement, can regress without curative treatment-like cutaneous lesions-although both multidisciplinary care and close follow-up should be implemented.

https://thejns.org/doi/abs/10.3171/2017.6.PEDS17113
\end{abstract}

KEY WORDS juvenile xanthogranuloma; intramedullary spinal cord lesion; spontaneous regression; spine; oncology

$\mathbf{J}$ UVENILE Xanthogranuloma (JXG) is a rare disease affecting both cutaneous and extracutaneous sites. ${ }^{3,7}$ It was previously considered to be endothelium derived and was thus named "nevoxanthoendothelioma." 18 It was initially classified under the family of non-Langerhans cell histiocytoses. ${ }^{3,7}$ However, a recently revised classification distinguishes cutaneous JXG, which belongs to the family of non-Langerhans cell histiocytoses, from extracutaneous or disseminated JXG, which is closer to Langerhans histiocytosis. ${ }^{9}$ Juvenile xanthogranuloma has a wide spectrum of clinical presentations and usually occurs before 5 years of age, mostly in the first 6 months of life. ${ }^{8}$ Frequently, JXG is revealed by a solitary cutaneous lesion-in up to $81 \%$ of cases. ${ }^{713}$ The lesion consists of a firm nodule and/or a papule or a raised plaque-like lesion with reddish to yellowish coloration.? Juvenile xanthogranuloma can also affect multiple skin sites, affect only extracutaneous sites, or involve both cutaneous and extracutaneous sites. ${ }^{7}$ Soft tissues, bones, viscera, eyes/orbits, oropharynx, and muscles are the most frequently affected extracutaneous sites. ${ }^{7,10}$ Relatively few cases of nervous system involvement by JXG have been reported in the literature, with an incidence ranging from $1 \%$ to $2.3 \%{ }^{7,8,13}$ The lesions can affect both central and peripheral nervous systems including the brain (53\%), spinal cord (intradural, extramedullary; $13 \%)$, trigeminal nerve ganglion (10\%), and spinal nerve roots $(5 \%) .{ }^{8}$ These lesions are benign and most cases regress spontaneously within months to years; ${ }^{9,21}$ this is particularly the case with skin lesions. No instance of an intramedullary spinal cord lesion of JXG has been reported 

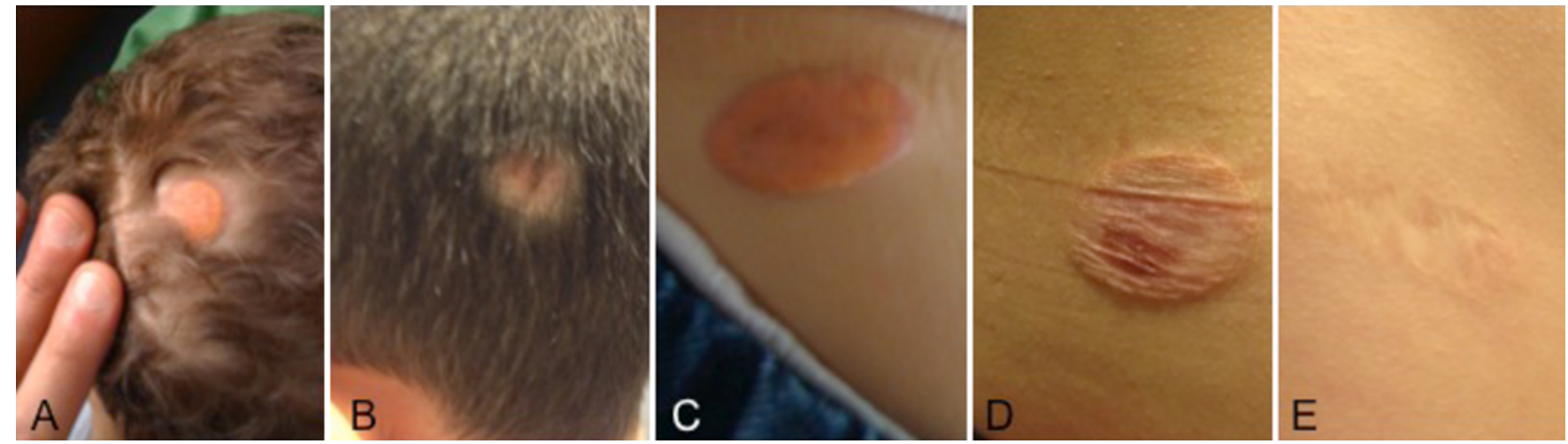

FIG. 1. Skin lesions of the scalp and gluteal region: initial aspect when the patient was 19 months old $(\mathbf{A}$ and $\mathbf{C})$ and spontaneous regression at 9 years old $(\mathbf{B}), 3$ years old $(\mathbf{D})$, and 9 years old $(\mathbf{E})$. Figure is available in color online only.

in the literature. We herein report a case of JXG with cutaneous, cerebral, and spinal involvement in a young boy with spontaneous regression of the lesions.

\section{Case Report}

History and Examination

The JXG diagnosis was suspected when the patient was 19 months old as his scalp and gluteal region (Fig. 1) had multiple yellow-red papulo-nodular skin lesions. Additionally, the boy presented with neurological symptoms including a delay in walking acquisition associated with distal paraparesis and urinary incontinence. Therefore, cerebral and spinal MRI was performed, which demonstrated the presence of 3 intracerebral nodular lesions located in the left temporal lobe (Fig. 2) as well as an intramedullary spinal cord lesion extending from T-8 to L-1 and associated with spinal cord malformation (high spinal cord; Fig. 3). The MRI showed the heterogeneous aspect of the intramedullary spinal cord lesion, isointense on T1weighted sequences with a cystic component hyperintense on T2-weighted sequences. The initial diagnosis of cutaneous JXG was histologically confirmed by biopsy of a nodular skin lesion (Fig. 4). The patient was then referred to our pediatric neurosurgical center, and we proposed to perform a biopsy of the unusual intramedullary spinal cord lesion.

\section{Operation}

To decrease the risk of postoperative spine deformity, the biopsy was performed via a posterior interlaminar approach at the low thoracic level (T10-11). Macroscopically, the spinal cord was enlarged and yellowish. Histologically, some large histiocytes were present in glial tissue-some of them with lipidized cytoplasm-along with some lymphocytes and eosinophils. These histiocytes expressed CD68, CD163, as well as factor XIIIa to a lesser extent (Fig. 5). These features confirmed the diagnosis of intramedullary spinal cord JXG.

\section{Postoperative Course}

Given the histopathological results, the unresectability
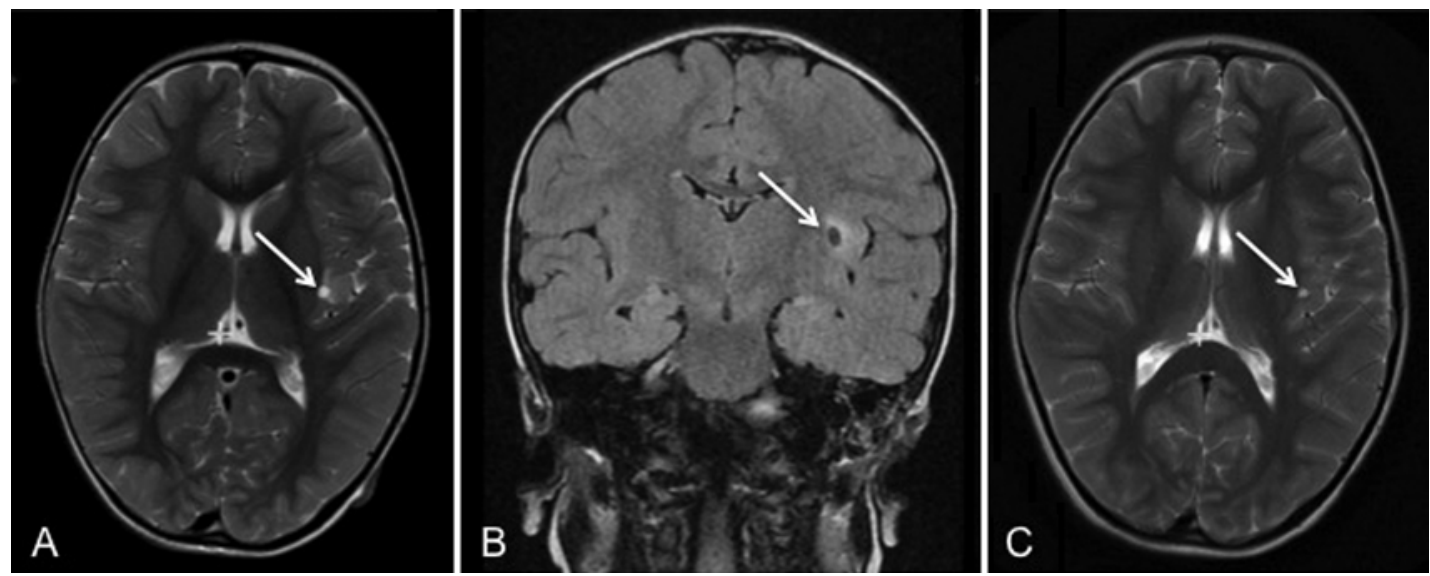

FIG. 2. Cerebral MR images showing regression of the intracerebral lesions (arrows) in the temporal lobe. Axial T2-weighted (A) and coronal FLAIR (B) sequences obtained when the patient was 28 months of age, showing a hyperintense lesion in the left insula and a hypointense lesion, respectively. Axial T2-weighted sequence $(\mathbf{C})$ obtained when the patient was 7 years old, showing regression of the hyperintense lesion. 

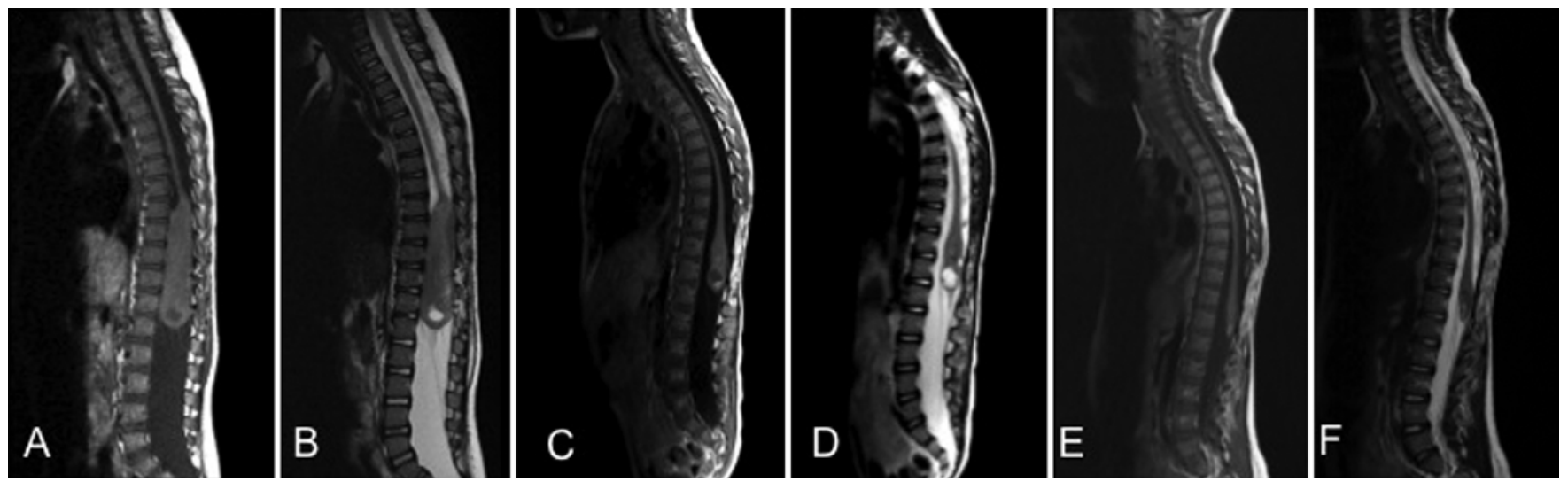

FIG. 3. Sagittal MR images obtained when the patient was 19 months old ( $A$ and $B$ ), 6 years old ( $C$ and $D$ ), and 9 years old ( $E$ and F), showing regression of the intramedullary spinal cord lesion initially extending from T-8 to L-1. Note the heterogeneous aspect of the lesion, isointense on T1-weighted sequences with minimal contrast enhancement $(A, C$, and $E)$ and a cystic component in the inferior part of the lesion, hyperintense on T2-weighted sequences ( $B$ and $D)$.

of the lesion, and the stability of the clinical signs over several months, we opted for close clinical and MRI follow-up. The boy started to walk at 33 months of age. We prescribed oxybutynin to limit the symptoms of urinary incontinence. Additionally, he underwent a motor rehabilitation program.

During follow-up, we observed gradual regression of the skin, cerebral, and intramedullary spinal cord lesions (Figs. 1-3) on MRI performed 6 months after surgery. After 9 years of follow-up, the patient is autonomous and can run and cycle. He wears night splints to correct his foot deformities, which have been stable for many years.

\section{Discussion}

Central and peripheral nervous system involvement by JXG is rare. Only 48 cases have been reported so far. Thirty-three cases involved the central nervous system, 12 cases involved the peripheral nervous system, and 3 cases involved intraosseous JXGs, whose primary origin was a vertebral body. ${ }^{1,8,16,19}$ Of the 12 patients with JXG lesions of the peripheral nervous system, 4 patients (33\%) had lesions in the trigeminal nerve, 3 patients $(25 \%)$ had lesions in a peripheral nerve, 3 patients $(25 \%)$ had lesions in a spinal nerve, and 2 patients (17\%) had lesions in the cauda equina. ${ }^{1,8}$ Of the 33 patients with a central nervous system lesion of JXG, 22 patients $(67 \%)$ had lesions in the brain, the cerebellum, or the posterior fossa and 1 patient (3\%) each had a lesion in the optic nerve, the sellar region, and the cavernous sinus. ${ }^{1,8,19}$ Involvement of the spinal cord by JXG was reported in 8 patients, and all lesions had an extramedullary location. ${ }^{8,16}$ Thus, our report is the first to describe an intramedullary spinal cord lesion of JXG associated with both brain and cutaneous lesions that all regressed spontaneously.

Central and peripheral nervous system involvement by JXG have distinct clinical presentations. Central nervous system lesions of JXG predominantly affect males. These patients are also affected at a younger age, compared to patients with peripheral nervous system lesions (5.75 vs 20.5 years, respectively). ${ }^{8}$ Additionally, central nervous system involvement by JXG is more frequently associated with cutaneous lesions than is peripheral nervous system involvement (46\% vs $10 \%){ }^{8}$

Diagnosis of JXG can prove difficult, especially in cases of solitary extracutaneous lesions, as clinical and macroscopically intraoperative findings can be nonspecific. Concerning differential diagnoses of JXG lesions involving the spinal cord, nerve roots, and peripheral nerves, nerve sheath tumors and meningioma should be considered. 5,6,8,11,14,15,20,22,23 Therefore, histopathological examination is the gold standard for diagnosing JXG ${ }^{8}$ However, the pathologist must rule out differential diagnoses when encountering xanthomatous lesions of the nervous system in children, such as nonneoplastic lesions (infarcts, demyelination, or infection), xanthomas associated with hyperlipidemia, and glial neoplasms (pleomorphic xanthoastrocytoma, granular cell astrocytoma, and oligodendroglioma). Other types of histiocytic disorders-mostly Langerhans cell histiocytosis-should also be ruled out. ${ }^{8}$ Histopathologically, JXGs are characterized by sheets of variably sized histiocytes with ample eosinophilic and vacuolated cytoplasm. The histiocytes are associated with varying numbers of admixed spindle cells and multinucleated giant cells including both Touton and foreign body type. Tumor cells express markers of hematogenous origin (CD45 [leukocyte common antigen]) as well as markers

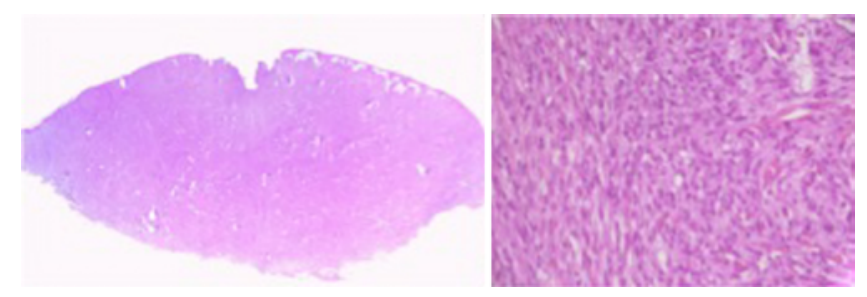

FIG. 4. Histopathological findings of the cutaneous lesion. Left: Nodular, rather well-circumscribed lesion located in the dermis and sparing the epidermis. Right: Medium-sized round or spindle-shaped, nonlipidized histiocytes. H \& E, original magnification $\times 25$ (left) and $\times 250$ (right). Figure is available in color online only. 

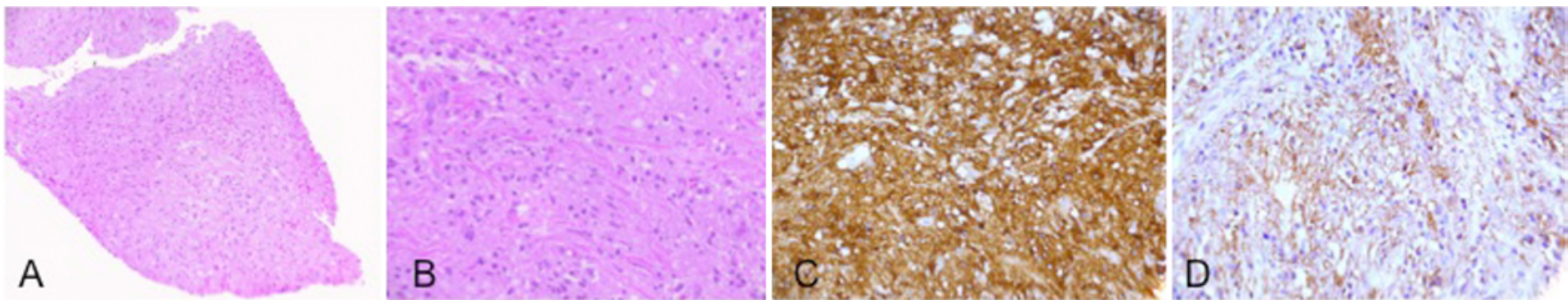

FIG. 5. Histopathological findings of the medullary biopsy. A: Presence of some large histiocytic cells mixed with glial tissue containing some axons. B: Presence of large histiocytic cells with vacuolated cytoplasm associated with some scattered eosinophils. C: Immunostaining for CD163. D: Immunostaining for factor XIIla. H \& E (A and B), original magnification $\times 200(A)$ and $\times 400(B-D)$. Figure is available in color online only.

of histiocytic dendrocytic differentiation (CD68, CD4, CD163, and factor XIIIa). ${ }^{4,8,17}$

Juvenile xanthogranuloma is often revealed by a solitary cutaneous lesion,, 13 which usually resolves spontaneously within 3 years. ${ }^{12}$ Systemic JXG is a rare disease and occurs in $4 \%-10 \%$ of patients with a solitary cutaneous lesion. ${ }^{7,10}$ It also frequently develops without skin lesions..$^{10}$ However, a solitary cutaneous lesion often predates systemic JXG lesions that involve the central nervous system. ${ }^{8,10}$ Therefore, when encountering cutaneous skin lesions of JXG, the clinician should be aware of possible associated extracutaneous lesions, including those of the central nervous system. He or she must also refer the patient for multidisciplinary care if some unusual symptoms occur. In the case of a cutaneous JXG lesion confirmed by the histopathological examination, the discovery of an intramedullary spinal cord lesion (with an MRI aspect similar to that in our case) should not necessarily lead to a biopsy. In fact, the indication for biopsy depends on the evolution of the clinical signs and the MRI aspects.

Management of nervous system lesions of JXG is highly heterogeneous in the literature and includes resection, chemotherapy, corticosteroids, or supportive care. ${ }^{1,8}$ Of the 33 patients reported with a JXG lesion of the central nervous system, 17 were treated only surgically, 6 had medical treatment (chemotherapy and/or corticotherapy), 2 had both surgical and medical treatments, 5 underwent chemotherapy and radiotherapy, 1 patient was observed, and treatment was unknown in 2 patients. ${ }^{1,8,16,19}$ Fifty-eight percent and $17 \%$ of the patients were disease free after surgery and after medical treatment (chemotherapy and/or corticotherapy), respectively. ${ }^{1,8,16,19}$ All the patients treated with chemotherapy and radiotherapy had an intracerebral lesion, and complete remission was never attained. ${ }^{8}$ Botella-Estrada et al. described a patient with multiple cutaneous lesions and asymptomatic JXG lesions of the cerebrum and cerebellum that were untreated and remained stable during follow-up. ${ }^{2}$ Recently, Miyake et al. have shown the possibility of spontaneous regression of a residual tumor of the occipital lobe after partial resection, which was indicated for epilepsy. ${ }^{19}$ Curative treatments reported in the literature do not seem more effective than supportive care in JXG. This highlights the potential for stability or for spontaneous resolution of JXG lesions. Moreover, curative treatments, in particular chemotherapy and radiotherapy, can lead to severe adverse effects in young children and should be reserved for progressive and/or life-threatening JXG lesions..$^{10}$ Although the choice of treatment should be guided by clinical signs, patient complaints, general health status, lesion locations, and safe resectability, clinicians should keep in mind that JXG-including intramedullary spinal cord lesions - may resolve spontaneously, as shown in our report.

\section{Conclusions}

We provided the first description of an intramedullary spinal cord lesion of JXG associated with cutaneous and cerebral lesions. Similar to those in other locations, the lesion resolved spontaneously within 7 years, without any medical or surgical treatment. However, if no treatment is provided, we would recommend close clinical and MRI follow-up for these patients.

\section{References}

1. Bhaisora KS, Jaiswal AK, Mehrotra A, Sahu RN, Srivastava A, Jaiswal S, et al: Solitary juvenile xanthogranuloma of the cervical spine in a child: a case report and review of literature. Asian J Neurosurg 10:57, 2015

2. Botella-Estrada R, Sanmartín O, Grau M, Alegre V, Mas C, Aliaga A: Juvenile xanthogranuloma with central nervous system involvement. Pediatr Dermatol 10:64-68, 1993

3. Burgdorf WHC, Zelger B: Die Nicht-Langerhans-Zell-Histiozytosen. Hautarzt 49:600-613, 1998

4. Burger P, Scheithauer B: AFIP Atlas of Tumor Pathology. Washington, DC: American Registry of Pathology, 2007,pp 519-539

5. Cao D, Ma J, Yang X, Xiao J: Solitary juvenile xanthogranuloma in the upper cervical spine: case report and review of the literatures. Eur Spine J 17 (Suppl 2):S318-S323, 2008

6. Castro-Gago M, Gómez-Lado C, Alvez F, Alonso A, Vieites B: Juvenile xanthogranuloma of the cauda equina. Pediatr Neurol 40:123-125, 2009

7. Dehner LP: Juvenile xanthogranulomas in the first two decades of life: a clinicopathologic study of 174 cases with cutaneous and extracutaneous manifestations. Am J Surg Pathol 27:579-593, 2003

8. Deisch JK, Patel R, Koral K, Cope-Yokoyama SD: Juvenile xanthogranulomas of the nervous system: A report of two cases and review of the literature. Neuropathology 33:3946, 2013

9. Emile JF, Abla O, Fraitag S, Horne A, Haroche J, Donadieu $\mathrm{J}$, et al: Revised classification of histiocytoses and neoplasms of the macrophage-dendritic cell lineages. Blood 127:26722681, 2016 
10. Freyer DR, Kennedy R, Bostrom BC, Kohut G, Dehner LP: Juvenile xanthogranuloma: forms of systemic disease and their clinical implications. J Pediatr 129:227-237, 1996

11. George DH, Scheithauer BW, Hilton DL, Fakhouri AJ, Kraus EW: Juvenile xanthogranuloma of peripheral nerve: a report of two cases. Am J Surg Pathol 25:521-526, 2001

12. Gianotti F, Caputo R: Histiocytic syndromes: a review. J Am Acad Dermatol 13:383-404, 1985

13. Janssen D, Harms D: Juvenile xanthogranuloma in childhood and adolescence: a clinicopathologic study of 129 patients from the Kiel Pediatric Tumor Registry. Am J Surg Pathol 29:21-28, 2005

14. Kimura H, Oka K, Nakayama Y, Tomonaga M: Xanthoma in Meckel's cave. A case report. Surg Neurol 35:317-320, 1991

15. Kitchen ND, Davies MS, Taylor W: Juvenile xanthogranuloma of nerve root origin. Br J Neurosurg 9:233-237, 1995

16. Konar S, Pandey P, Yasha TC: Solitary juvenile xanthogranuloma in cervical spine: case report and review of literature. Turk Neurosurg 24:102-107, 2014

17. Kontos G, Borooah S, Khan A, Fleck BW, Coupland SE: The epidemiology, clinical characteristics, histopathology and management of juvenile- and adult-onset corneoscleral limbus xanthogranuloma. Graefes Arch Clin Exp Ophthalmol 254:413-420, 2016

18. McDonagh JER: A contribution to our knowledge of the naevo-xantho-endotheliomata. Br J Dermatol 24:85-99, 1912

19. Miyake Y, Ito S, Tanaka M, Tanaka Y: Spontaneous regression of infantile dural-based non-Langerhans cell histiocytosis after surgery: case report. J Neurosurg Pediatr 15:372-379, 2015

20. Nakasu S, Tsuji A, Fuse I, Hirai H: Intracranial solitary juvenile xanthogranuloma successfully treated with stereotactic radiosurgery. J Neurooncol 84:99-102, 2007
21. Pajaziti L, Hapçiu SR, Pajaziti A: Juvenile xanthogranuloma: a case report and review of the literature. BMC Res Notes 7:174, 2014

22. Patil PG, Cummings TJ, Nunley JA, Friedman AH: Xanthogranuloma of the sciatic nerve: case report. Neurosurgery 55:1435, 2004

23. Paulus W, Kirchner T, Ott MM, Kühl J, Warmuth-Metz M, Sörensen N, et al: Histiocytic tumor of Meckel's cave. An intracranial equivalent of juvenile xanthogranuloma of the skin. Am J Surg Pathol 16:76-83, 1992

\section{Disclosures}

The authors report no conflict of interest concerning the materials or methods used in this study or the findings specified in this paper.

\section{Author Contributions}

Conception and design: Morice. Acquisition of data: Morice. Analysis and interpretation of data: Fraitag, Miquel, Sainte Rose. Drafting the article: Morice. Critically revising the article: Puget, Fraitag. Reviewed submitted version of manuscript: Puget. Approved the final version of the manuscript on behalf of all authors: Puget. Care of patient: Puget, Sainte Rose.

\section{Correspondence}

Stéphanie Puget, Department of Pediatric Neurosurgery, Hôpital Necker, Sorbonne Paris Cité, 149 rue de Sèvres, Paris 75015, France. email: stephanie.puget@gmail.com. 